Review

\title{
Multidrug-Resistant Bacterial Infections before and after Liver Transplantation
}

Alberto Ferrarese, Marco Senzolo, Patrizia Burra *

Multivisceral Transplant Unit, Department of Surgery, Oncology and Gastroenterology, Padua University Hospital, via Giustiniani, 2, 35128 Padua, Italy; E-Mails: alberto.ferrarese17@gmail.com; marcosenzolo@hotmail.com; $\underline{\text { burra@unipd.it }}$

*Correspondence: Patrizia Burra; E-Mail: burra@unipd.it

Academic Editor: Yasuhiko Sugawara

Special Issue: Infections in Liver Transplantation

OBM Transplantation

2020, volume 4, issue 2

doi:10.21926/obm.transplant.2002110
Received: March 29, 2020

Accepted: May 11, 2020

Published: May 18, 2020

\begin{abstract}
Bacterial infection represents a common event in the natural history of cirrhosis, especially in patients with end-stage liver disease. An episode of bacterial infection may significantly modify a patient's outcome in the setting of liver transplantation (LT) by impairing hepatic and extrahepatic organ functions, both in pre-operative and post-operative phases. Infections caused by multidrug-resistant organisms (MDRO) are increasing in cirrhosis due to high antibiotic exposure and hospitalizations. This issue is particularly important in the LT setting, where MDRO infections occurring before surgery and during the early days after surgery might significantly impair graft function and patient outcome. This review summarizes the recently published findings on the occurrence of MDRO infection before and after LT, and new perspectives for its prevention and treatment.
\end{abstract}

\section{Keywords}

Liver transplantation; multidrug-resistant bacteria; bacterial infection; sepsis

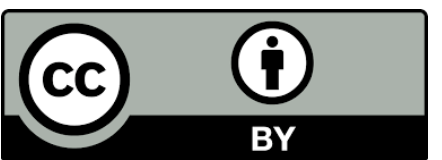

(C) 2020 by the author. This is an open access article distributed under the conditions of the Creative Commons by Attribution License, which permits unrestricted use, distribution, and reproduction in any medium or format, provided the original work is correctly cited. 


\section{Bacterial Infection in Cirrhosis: An Evolving Scenario}

The liver is actively involved in the inflammatory response against bacteria and plays a central role in the regulation of immune defense [1]. This pro- and anti-inflammatory balance is often impaired or even lost in the presence of acute or chronic liver diseases.

Cirrhosis is characterized by an immunocompromised state and exhaustion of immune response to pathogens due to immunodeficiency and persistent systemic inflammation [2].

Therefore, patients with cirrhosis are prone to develop a bacterial infection (BI) during the natural history of the disease [3]. Prevalence of BI ranges from $20 \%$ to $30 \%$ in hospitalized patients, with the rate being significantly associated with the severity of the underlying condition [4, 5]. The occurrence of $\mathrm{BI}$ further impairs circulatory dysfunction and increase portal hypertension, accompanied by splanchnic vasodilation and hypoperfusion of extrahepatic organ(s) $[6,7]$. As a consequence, $\mathrm{BI}$ is a common trigger for the development of acute-on-chronic liver failure (ACLF) $[8,9]$, which is associated with short-term mortality [10].

In the past few decades, clinical practice in the field of hepatology has changed dramatically as a consequence of the implementation of liver transplant (LT) programs. Cirrhotic patients are more frequently admitted to the ICU nowadays. They undergo several diagnostic and therapeutic invasive procedures and are repeatedly in contact with healthcare facilities. Such progress in the care of cirrhosis has led to an increasing rate of nosocomial/healthcare-associated $\mathrm{BI}$, and ultimately to an alarming spread of infections caused by multidrug-resistant organisms (MDRO) [11-13]. Epidemiology, risk factors, and clinical features of resistant strains-such as vancomycinresistant enterococci (VRE), methicillin-resistant staphylococci, extended-spectrum $\beta$-lactamase enterobacterales (ESBLE) and carbapenem-resistant enterobacterales (CRE)-have been widely reported in cirrhosis [14-17]. Notably, the latter group encompasses bacteria with multiple molecular resistance mechanisms, including the ability to produce carbapenemases (which are further categorized according to their molecular structure/active sites) [18].

The occurrence of MDRO infection in the setting of LT is a detrimental event, as it can significantly affect the patient's short-term outcome. This review provides new perspectives in this area, with a focus on the outcome of the occurrence of MDRO infection immediately before or after surgery.

\section{Multidrug-Resistant Bacterial Infection Occurring before LT}

The prevalence of MDRO infections in cirrhosis is variable across studies. Several factors may explain this heterogeneity. Firstly, even though an MDRO is commonly considered according to resistance to at least one agent in three different antibiotic classes [19], different definitions have been suggested in studies on MDRO and cirrhosis. Secondly, in many cases, the terms of systemic infection and colonization have been used interchangeably. However, colonization implies the presence of a microorganism in a host, but without any overt clinical expression or detectable immune reaction. Conversely, the term infection implies the successful multiplication of a bacterium within a host, triggering an immune response $[20,21]$. These definitions have been widely accepted in the setting of cirrhosis and solid organ transplantation [22-24]. Thirdly, since the risk of $\mathrm{BI}$ is strongly associated with the severity of liver disease and patients' characteristics (e.g., the setting and timing of evaluation), the studies cannot be compared to each other as they 
include heterogeneous cohorts of cirrhotics. Lastly, many BIs in cirrhosis turn out culture-negative (e.g., pneumonia, spontaneous bacterial peritonitis); thus, the prevalence of MDRO infections could be underestimated.

Summarizing the available literature, MDRO are responsible for $20-30 \%$ of culture-positive infections in patients with cirrhosis. A multicenter study conducted in Italy [16] reported $27 \%$ prevalence of MDRO infections in 395 patients, mainly due to gram-negative rods (ESBLE and CRE). Another study from Greece [25], examined only the prevalence of spontaneous bacterial peritonitis, and reported lower prevalence (19\%). In a study in Egypt [26], which considered only cirrhotic patients in the ICU, there was $52.6 \%$ prevalence of MDRO pathogens in culture-positive infections, mainly methicillin-resistant staphylococci, ESBLE, and CRE.

A recent prospective, observational, multicenter study [15], which included forty-six centers worldwide, reported that MDRO accounted for $34 \%$ of culture-positive infections. This study highlighted relevant geographic and epidemiological differences and reinforced the link between MDRO infections and decompensated liver function, prior use of systemic antibiotics, invasive procedures, and exposure to healthcare workers. In another landmark study, which considered only patients with decompensated cirrhosis and ACLF, Fernandez et al. [13] reported similar prevalence of MDRO infections (29\%) at a rate equal to $28 \%, 23.5 \%$ and $20.7 \%$ for bloodstream infections, pneumonia, and urinary tract infections, respectively. As expected, the resolution rate of MDRO infections was significantly lower than that of non-MDRO infections; furthermore, the former group led to a higher prevalence of severe sepsis/shock and ACLF, increasing the rate of short-term mortality.

Few epidemiological data are available in the setting of LT. We have previously demonstrated a $24.2 \%$ rate of MDRO-related BI in a large cohort of 876 patients enlisted for LT between 2006 and 2014 at our center [27]; notably, CRE and methicillin-resistant staphylococci were the commonest strains of MDRO. With regard to the outcome, BI occurring while awaiting LT or during the days before surgery has been associated with controversial results. A multicenter study in the US [28] clearly showed that the risk of being delisted from the LT waiting list within six months was much higher in infected patients with cirrhosis (42\%) than in non-infected wait-listed patients, even in the case where a sub-analysis on MDRO strains was not yet reported. Moreover, a recent study on the Eurotransplant database by Friedrich et al. [29] showed that twenty-three LT candidates with pre-LT MDRO infection had significantly higher chances of being delisted from the waiting list than patients without infection, since the occurrence of MDRO infection led to a two-fold mortality risk while awaiting transplantation. In the above-mentioned study conducted at our center [27], we demonstrated that for patients in the waiting list for $\mathrm{LT}, \mathrm{BI}$ was an independent predictor of mortality, but patients who recovered from $\mathrm{BI}$ and readmitted to the waiting list after thirty days had a good probability of receiving LT, and their mortality risk was similar to patients without infection.

Considering the post-LT phase, several studies have shown that pre-LT MDRO infections might significantly affect post-LT survival [30, 31]. Conversely, a few other authors did not find differences in the early postoperative outcome if pre-LT infections were adequately treated [32, 33]. In a study in France conducted on the sickest ACLF patients admitted to the ICU, uncontrolled sepsis remained a contraindication for LT, whereas treated sepsis was not a reason to rule out $a$ priori the intervention [34]; nevertheless, no cases of MDRO infection were reported. Thus, we can 
hypothesize that the control of a MDRO related sepsis might be very difficult, and it would take much more time than a non-MDRO infection in such an LT setting.

An MDRO colonization represents an important issue while awaiting transplantation. For the above-mentioned factors, such colonization occurs more frequently in the sickest, hospitalized patients with cirrhosis. Thus, the risk of a postoperative MDRO systemic infection should be considered case-by-case and carefully balanced with the risk of short-term mortality as in a case without a transplant. A recently published consensus conference on colonization in the solid organ transplant setting [22] confirmed the association between ESBLE colonization and worsened postoperative outcome, but the panel did not consider it as a contraindication for transplantation. Indeed, pre-operative specific antibiotic prophylaxis and early institution of a targeted antibiotic treatment after surgery might reduce mortality and graft loss in such patients.

An MDRO colonization due to CRE and VRE, while awaiting $L T$, represents an even more difficult scenario due to the high risk of postoperative onset of a systemic infection, and the relatively low number of antibiotics available for treatment [35-38]. Nonetheless, to date, there are no data to firmly contraindicate transplantation of patients colonized with CRE and VRE [22].

Taken together, these data suggest that the prevalence of MDRO infections is increasing dramatically in patients with cirrhosis (with the current rate ranging from 20 to $30 \%$ ), and is more common among the sickest and hospitalized patients. MDRO infection is often associated with poor survival and further impairment of hepatic and extrahepatic organs, leading to ACLF. Since an MDRO infection occurring early before LT might influence the postoperative course, an effective treatment strategy is advisable before considering the patient suitable for LT. MDRO colonization occurring in the pre-LT phase does not firmly contraindicate transplantation; however, since it has been associated with a higher risk of postoperative complications (including graft loss), the decision to proceed with LT should be carefully weighed, especially for the sickest patients with CRE or VRE colonization.

\section{Multidrug Bacterial Infection Early after Liver Transplantation}

The prevalence of $\mathrm{BI}$ in the early phase after $\mathrm{LT}$ ranges from $30 \%$ to $60 \%$, with surgical site infections, catheter-related bloodstream infections, and ventilator-associated pneumonia being the commonest sources [39-41]. The occurrence of a BI in the early postoperative days often leads to impairment of graft function and delay in the introduction of immunosuppression at an effective dosage; furthermore, it significantly influences the functioning of an extrahepatic organ (s), leading to a prolonged ICU stay and impaired short-term graft function [42, 43]. The occurrence of an MDRO infection has been associated with a worsened post-LT survival rate [44, 45].

An MDRO infection may occur in the post-LT phase through any of the three important mechanisms: post-LT infection from a pre-LT infection, post-LT colonization, or donor-derived infection.

Pre-LT colonization has been considered a risk factor for post-LT MDRO colonization and subsequent infection, especially by CRE strains [46]. Giannella et al. [36] demonstrated in a prospective group of 237 LT patients that CRE colonization before LT was an independent risk factor for post-LT CRE infection. The same group [35] confirmed the data evaluating the occurrence of CRE systemic infections in a larger cohort of 553 LT recipients. At multivariate 
analysis, CRE colonization at LT was an independent risk factor for the occurrence of CRE infection within one year after LT, along with post-LT colonization, combined transplant, prolonged mechanical ventilation, re-intervention, and rejection. Another prospective study by Freire et al. [47] confirmed that both pre- and post-LT CRE colonization were significant risk factors for developing a CRE infection early after LT (OR 12.75; 95\% Cl 5.5-29; OR 4.14, 95\% Cl 1.93-8.90, respectively), along with a MELD score $>32$ at $L T$, combined transplant, reoperation, and post-LT need for dialysis. Similar results were observed for MDRO infections caused by carbapenemresistant Acinetobacter baumannii [48]. Notably, patients who experienced such an infection were more prone to colonization before surgery and displayed a significant postoperative mortality rate (equal to $46 \%$ within two months of surgery).

Robust data are also available for gram-positive MDRO pathogens. A meta-analysis of twentythree studies on solid organ transplantations (seventeen on LT) [49] confirmed that pre-transplant and post-transplant methicillin-resistant staphylococcal colonization increased the risk of posttransplant systemic infection (pooled risk ratio: 5.51; 95\% $\mathrm{Cl} 2.36-12.90$ and 10.56 ; $95 \% \mathrm{Cl} 5.58-$ 19.95, respectively); similarly, pre-transplant and post-transplant VRE colonization was also associated with a significant risk of VRE infection (RR 6.65; $95 \% \mathrm{Cl} 2.54-17.41$ and $\mathrm{RR} 7.93 ; 95 \% \mathrm{Cl}$ 2.36-26.67, respectively).

Donor-derived infections (DDI) caused by MDRO pathogens have been increasingly reported to have a significant impact on the recipients' outcome [44]. Deceased donors are often critically ill patients admitted to the ICU and have several risk factors for developing or acquiring MDRO colonization or infection. Lewis et al. [50] in 2016 summarized the available literature in this field, showing that around $50 \%$ of solid transplant recipients who received a graft from a donor with MDRO infection, subsequently acquired a DDI. Furthermore, gram-negative MDRO infections were associated with a worse prognosis than gram-positive ones ( $41 \%$ vs. $14 \%$, respectively), but the low sample size might have influenced the results.

Two recent studies corroborate these findings. The first study conducted in the US [51] reported $15 \%$ prevalence of MDRO-related infections in 440 solid organ donors, with Hepatitis C, dialysis, prior hematopoietic cell transplant, and exposure to antibiotics with a narrow Gramnegative spectrum acting as independent predictors. The second study carried out in Italy [52] investigated the prevalence of MDRO infections in 759 deceased donors. The authors showed that screening for MDRO was performed in $99 \%$ of patients during the observation period, but results were not available at the time of transplantation in a significant percentage of patients (20\%). There were 36 cases of CRE infection among donors, and most of them were not discarded since culture positivization occurred after transplantation. Considering the early post-transplant phase, when the transplant was not related to the site of CRE isolation, 90-d post-transplant mortality was low (8.3\%), without any association with sepsis or CRE infection. When transplants were performed in the presence of CRE in the same site and/or in blood, mortality was higher (23.1\%); in two cases (one lung and one liver), death was associated with early post-transplant CRE sepsis due to the same CRE species as found in donor.

Therefore, since absolute prevention of donor-recipient transmission of bacterial infections is not always feasible, strict microbial surveillance of the donors is of utmost importance, especially for donors with prolonged ICU stay, fever, and suspicion of an ongoing clinical infection. In the diagnosis of DDI, an early administration of targeted antibiotic therapy is mandatory, even if preemptive therapy or prophylaxis fails to prevent DDI in many cases [44]. 
Taken together, these data suggest that pre-LT and post-LT MDRO colonization represent a major risk factor for post-LT MDRO infection, especially in the case of CRE. The severity of the liver disease at the time of transplantation, post-LT complications as well as longer ICU stay represent other risk factors for MDRO-related infections. Although rare, a donor-derived MDRO infection represents a serious complication, which can significantly impair graft function and patient outcome in the early post-transplantation phase.

\section{Strategies to Prevent and Treat Multidrug Bacterial Infections in the Setting of Liver Transplantation}

The principles of antibiotic treatment of MDRO infections go beyond the aim of this manuscript. Furthermore, robust data on the safety and effectiveness of novel antibiotics, such as the combinations of carbapenems for CRE or combination of ceftaroline, dalbavancin, and delafloxacin for methicillin-resistant staphylococci, are yet to come in the liver transplantation setting [53, 54].

Nevertheless, some information may be of importance for the prevention and treatment of MDRO infections in the LT setting.

Since MDRO infections often develop in the sickest, hospitalized patients with cirrhosis, organ dysfunction, and prior non-MDRO bacterial infections, the antibiotic stewardship remains paramount for preventing the emergence of resistant rods before $L T[12,55]$. This might include an accurate evaluation of the local epidemiological trends of bacterial infections using a multidisciplinary approach encompassing infectious disease specialists, anesthesiologists, transplant surgeons, pharmacists and hepatologists, and tailored treatment algorithms [56, 57].

Furthermore, active surveillance for MDRO colonization both before and after LT should be universally performed, in order to institute an adequate prophylactic or pre-emptive antibiotic therapy in the early postoperative phase, or whenever needed.

The role of intestinal decontamination in cirrhotics has been under debate. A meta-analysis of four randomized studies published between 1994 and 2002 by Safdar et al. [58] showed the benefit of oral decontamination in reducing the incidence of only gram-negative infections in the LT setting (84\% reduction), whereas it had no effect on overall infections. These results have been confirmed in a further meta-analysis by Gurusamy et al. [59], who did not find significant differences in mortality or post-LT infections when comparing oral decontamination with placebo, prebiotics and probiotics. Notably, an observational study by Katchman et al. [60] focused on the role of intestinal decontamination in the setting of a living donor LT, where surgery could be more effectively planned. The authors began administering oral colistin plus gentamicin three days before surgery until the day of discharge or up to a maximum of 14 days after surgery but they did not find a decrease in post-LT infection rate $(42.7 \%$ vs. $46.8 \%)$. In this study, however, oral decontamination was administered to patients undergoing LT irrespective of the presence/absence of MDRO colonization before surgery.

Fecal microbiota transplantation (FMT) has been proposed as a novel technique for intestinal decontamination in the sickest patients. This therapeutic approach was originally considered for the treatment of refractory/recurrent Clostridioides difficile infection, with good results in terms of safety and effectiveness. It is also useful for immunocompromised patients [61].

In the field of MDRO colonization, the available data were collected from small cohorts of patients, who had heterogeneous underlying conditions $[62,63]$. A recent multicentric study 
showed a $70 \%$ de-colonization rate using FMT in a small cohort of patients undergoing allogeneic hematopoietic stem cell transplantation without serious adverse events [64].

Safety and effectiveness of FMT are two major issues found in the sickest patients with cirrhosis experiencing MDRO colonization. Preliminary data coming from studies on FMT in the treatment of hepatic encephalopathy showed promising results with only a few side effects, also, in the longterm follow-up $[65,66]$. However, further studies are warranted before considering FMT as a wellestablished and safe option for intestinal decontamination in such patients [67].

\section{Conclusions}

MDRO-related infections represent an emerging challenge in Hepatology. According to the available data, these infections significantly impair survival in both decompensated cirrhotics awaiting LT and early postoperative phase cirrhotics. Since pre-LT and post-LT colonization was an important risk factor for post-surgical invasive infection, standardized surveillance strategies for colonization are mandatory. Careful screening of the highest risk organ donors is advisable in order to institute early and effective treatment strategies after donor-to-recipient MDRO transmission is established. Robust data on the safety and effectiveness of new antimicrobial combinations, as well as FMT for the treatment of MDRO infections and colonization in this specific setting, are urgently needed.

\section{Abbreviations}

ACLF: acute-on-chronic liver failure; BI: bacterial infection; CRE: carbapenems resistant enterobacterales; DDI: donor-derived infection; ESBLE: extended-spectrum beta-lactamase enterobacterales; FMT: fecal microbiota transplantation; LT: liver transplantation; MDRO: multidrug-resistant organism; VRE: vancomycin-resistant enterococci.

\section{Acknowledgments}

None to declare.

\section{Author Contributions}

$\mathrm{AF}, \mathrm{PB}, \mathrm{MS}$ participated in research design, performance of the research and writing of the manuscript.

\section{Funding}

No organization(s) nor foundation(s) funded this research.

\section{Competing Interests}

The authors have declared that no competing interests exist. 


\section{References}

1. Strnad P, Tacke F, Koch A, Trautwein C. Liver - guardian, modifier and target of sepsis. Nat Rev Gastroenterol Hepatol. 2017; 14: 55-66.

2. Albillos A, Lario M, Álvarez-Mon M. Cirrhosis-associated immune dysfunction: Distinctive features and clinical relevance. J Hepatol. 2014; 61: 1385-1396.

3. D'Amico G, Morabito A, D'Amico M, Pasta L, Malizia G, Rebora P, et al. Clinical states of cirrhosis and competing risks. J Hepatol. 2018; 68: 563-576.

4. Dionigi E, Garcovich M, Borzio M, Leandro G, Majumdar A, Tsami A, et al. Bacterial infections change natural history of cirrhosis irrespective of liver disease severity. Am J Gastroenterol. 2017; 112: 588-596.

5. Ferrarese A, Zanetto A, Becchetti C, Sciarrone SS, Shalaby S, Germani G, et al. Management of bacterial infection in the liver transplant candidate. World J Hepatol. 2018; 10: 222-230.

6. Moreau R, Hadengue A, Soupison T, Kirstetter P, Mamzer MF, Vanjak D, et al. Septic shock in patients with cirrhosis: Hemodynamic and metabolic characteristics and intensive care unit outcome. Crit Care Med. 1992; 20: 746-750.

7. Thalheimer U, Triantos CK, Samonakis DN, Patch D, Burroughs AK. Infection, coagulation, and variceal bleeding in cirrhosis. Gut. 2005; 54: 556-563.

8. Moreau R, Jalan R, Gines P, Pavesi M, Angeli P, Cordoba J, et al. Acute-on-chronic liver failure is a distinct syndrome that develops in patients with acute decompensation of cirrhosis. Gastroenterology. 2013; 144: 1426-1437, 1437.e1-9.

9. Fernández J, Acevedo J, Wiest R, Gustot T, Amoros A, Deulofeu C, et al. Bacterial and fungal infections in acute-on-chronic liver failure: Prevalence, characteristics and impact on prognosis. Gut. 2018; 67: 1870-1880.

10. Arvaniti V, D'Amico G, Fede G, Manousou P, Tsochatzis E, Pleguezuelo M, et al. Infections in patients with cirrhosis increase mortality four-fold and should be used in determining prognosis. Gastroenterology. 2010; 139: 1246-1256, 1256.e1-5.

11. Fernández J, Navasa M, Gómez J, Colmenero J, Vila J, Arroyo V, et al. Bacterial infections in cirrhosis: Epidemiological changes with invasive procedures and norfloxacin prophylaxis. Hepatology. 2002; 35: 140-148.

12. Fernández J, Bert F, Nicolas-Chanoine $\mathrm{MH}$. The challenges of multi-drug-resistance in hepatology. J Hepatol. 2016; 65: 1043-1054.

13. Fernández J, Prado V, Trebicka J, Amoros A, Gustot T, Wiest R, et al. Multidrug-resistant bacterial infections in patients with decompensated cirrhosis and with acute-on-chronic liver failure in Europe. J Hepatol. 2019; 70: 398-411.

14. Hand J, Patel G. Multidrug-resistant organisms in liver transplant: Mitigating risk and managing infections. Liver Transpl. 2016; 22: 1143-1153.

15. Piano S, Singh V, Caraceni P, Maiwall R, Alessandria C, Fernandez J, et al. Epidemiology and effects of bacterial infections in patients with cirrhosis worldwide. Gastroenterology. 2019; 156: 1368-1380.e1310.

16. Salerno F, Borzio M, Pedicino C, Simonetti R, Rossini A, Boccia S, et al. The impact of infection by multidrug-resistant agents in patients with cirrhosis. A multicenter prospective study. Liver Int. 2017; 37: 71-79. 
17. Merli M, Lucidi C, Di Gregorio V, Falcone M, Giannelli V, Lattanzi B, et al. The spread of multi drug resistant infections is leading to an increase in the empirical antibiotic treatment failure in cirrhosis: A prospective survey. PLoS One. 2015; 10: e0127448.

18. Goodman KE, Simner PJ, Tamma PD, Milstone AM. Infection control implications of heterogeneous resistance mechanisms in carbapenem-resistant enterobacteriaceae (CRE). Expert Rev Anti Infect Ther. 2016; 14: 95-108.

19. Magiorakos AP, Srinivasan A, Carey RB, Carmeli Y, Falagas ME, Giske CG, et al. Multidrugresistant, extensively drug-resistant and pandrug-resistant bacteria: An international expert proposal for interim standard definitions for acquired resistance. Clin Microbiol Infect. 2012; 18: 268-281.

20. Healthcare associated infections: Centers Disease Control Prevention; [Available from: www.cdc.gov.]

21. Jarvis W. Bennett \& brachman's hospital infections: Lippincott Williams \& Wilkins; 2013.

22. Aguado JM, Silva JT, Fernández-Ruiz M, Cordero E, Fortún J, Gudiol C, et al. Management of multidrug resistant Gram-negative bacilli infections in solid organ transplant recipients: SET/GESITRA-SEIMC/REIPI recommendations. Transplant Rev (Orlando). 2018; 32: 36-57.

23. Tacconelli E, Mazzaferri F, de Smet AM, Bragantini D, Eggimann P, Huttner BD, et al. Escmideucic clinical guidelines on decolonization of multidrug-resistant Gram-negative bacteria carriers. Clin Microbiol Infect. 2019; 25: 807-817.

24. Jalan R, Fernandez J, Wiest R, Schnabl B, Moreau R, Angeli P, et al. Bacterial infections in cirrhosis: A position statement based on the easl special conference 2013. J Hepatol. 2014; 60: 1310-1324.

25. Alexopoulou A, Papadopoulos N, Eliopoulos DG, Alexaki A, Tsiriga A, Toutouza M, et al. Increasing frequency of Gram-positive cocci and Gram-negative multidrug-resistant bacteria in spontaneous bacterial peritonitis. Liver Int. 2013; 33: 975-981.

26. Hassan EA, Elsherbiny NM, Abd El-Rehim AS, Soliman AMA, Ahmed AO. Health careassociated infections in pre-transplant liver intensive care unit: Perspectives and challenges. J Infect Public Health. 2018; 11: 398-404.

27. Ferrarese A, Vitale A, Sgarabotto D, Russo FP, Germani G, Gambato M, et al. Outcome of a first episode of bacterial infection in candidates for liver transplantation. Liver Transpl. 2019; 25: 1187-1197.

28. Reddy KR, O'Leary JG, Kamath PS, Fallon MB, Biggins SW, Wong F, et al. High risk of delisting or death in liver transplant candidates following infections: Results from the North American consortium for the study of end-stage liver disease. Liver Transpl. 2015; 21: 881-888.

29. Friedrich K, Krempl J, Schamoni S, Hippchen T, Pfeiffenberger J, Rupp C, et al. Multidrugresistant bacteria and disease progression in patients with end-stage liver disease and after liver transplantation. J Gastrointestin Liver Dis. 2019; 28: 303-310.

30. Levesque E, Winter A, Noorah Z, Daurès JP, Landais P, Feray C, et al. Impact of acute-onchronic liver failure on 90-day mortality following a first liver transplantation. Liver Int. 2017; 37: 684-693.

31. Huebener P, Sterneck MR, Bangert K, Drolz A, Lohse AW, Kluge S, et al. Stabilisation of acuteon-chronic liver failure patients before liver transplantation predicts post-transplant survival. Aliment Pharmacol Ther. 2018; 47: 1502-1510. 
32. Bertuzzo VR, Giannella M, Cucchetti A, Pinna AD, Grossi A, Ravaioli $M$, et al. Impact of preoperative infection on outcome after liver transplantation. Br J Surg. 2017; 104: e172e181.

33. Sun HY, Cacciarelli TV, Singh N. Impact of pretransplant infections on clinical outcomes of liver transplant recipients. Liver Transpl. 2010; 16: 222-228.

34. Artru F, Louvet A, Ruiz I, Levesque E, Labreuche J, Ursic-Bedoya J, et al. Liver transplantation in the most severely ill cirrhotic patients: A multicenter study in acute-on-chronic liver failure grade 3. J Hepatol. 2017; 67: 708-715.

35. Giannella M, Bartoletti M, Campoli C, Rinaldi M, Coladonato S, Pascale R, et al. The impact of carbapenemase-producing enterobacteriaceae colonization on infection risk after liver transplantation: A prospective observational cohort study. Clin Microbiol Infect. 2019; 25: 1525-1531.

36. Giannella M, Bartoletti M, Morelli MC, Tedeschi S, Cristini F, Tumietto F, et al. Risk factors for infection with carbapenem-resistant klebsiella pneumoniae after liver transplantation: The importance of pre- and posttransplant colonization. Am J Transplant. 2015; 15: 1708-1715.

37. Nellore A, Huprikar S, Practice AICo. Vancomycin-resistant enterococcus in solid organ transplant recipients: Guidelines from the American Society of Transplantation Infectious Diseases Community of Practice. Clin Transplant. 2019; 33: e13549.

38. Russell DL, Flood A, Zaroda TE, Acosta C, Riley MM, Busuttil RW, et al. Outcomes of colonization with MRSA and VRE among liver transplant candidates and recipients. Am J Transplant. 2008; 8: 1737-1743.

39. Fishman JA. Infection in solid-organ transplant recipients. N Engl J Med. 2007; 357: 2601-2614.

40. Romero FA, Razonable RR. Infections in liver transplant recipients. World J Hepatol. 2011; 3: 83-92.

41. Gagliotti C, Morsillo F, Moro ML, Masiero L, Procaccio F, Vespasiano F, et al. Infections in liver and lung transplant recipients: A national prospective cohort. Eur J Clin Microbiol Infect Dis. 2018; 37: 399-407.

42. Pedersen $M$, Seetharam A. Infections after orthotopic liver transplantation. J Clin Exp Hepatol. 2014; 4: 347-360.

43. Saner FH, Akkiz H, Canbay A. Infectious complications in the early postoperative period in liver transplant patients. Minerva Gastroenterol Dietol. 2010; 56: 355-365.

44. Bartoletti M, Giannella M, Tedeschi S, Viale P. Multidrug-resistant bacterial infections in solid organ transplant candidates and recipients. Infect Dis Clin North Am. 2018; 32: 551-580.

45. Kalpoe JS, Sonnenberg E, Factor SH, del Rio Martin J, Schiano T, Patel G, et al. Mortality associated with carbapenem-resistant klebsiella pneumoniae infections in liver transplant recipients. Liver Transpl. 2012; 18: 468-474.

46. Macesic N, Gomez-Simmonds A, Sullivan SB, Giddins MJ, Ferguson SA, Korakavi G, et al. Genomic surveillance reveals diversity of multidrug-resistant organism colonization and infection: A prospective cohort study in liver transplant recipients. Clin Infect Dis. 2018; 67: 905-912.

47. Freire MP, Oshiro IC, Pierrotti LC, Bonazzi PR, de Oliveira LM, Song AT, et al. Carbapenemresistant enterobacteriaceae acquired before liver transplantation: Impact on recipient outcomes. Transplantation. 2017; 101: 811-820. 
48. Freire MP, Pierrotti LC, Oshiro IC, Bonazzi PR, Oliveira LM, Machado AS, et al. Carbapenemresistant acinetobacter baumannii acquired before liver transplantation: Impact on recipient outcomes. Liver Transpl. 2016; 22: 615-626.

49. Ziakas PD, Pliakos EE, Zervou FN, Knoll BM, Rice LB, Mylonakis E. MRSA and VRE colonization in solid organ transplantation: A meta-analysis of published studies. Am J Transplant. 2014; 14: $1887-1894$.

50. Lewis JD, Sifri CD. Multidrug-resistant bacterial donor-derived infections in solid organ transplantation. Curr Infect Dis Rep. 2016; 18: 18.

51. Anesi JA, Blumberg EA, Han JH, Lee DH, Clauss H, Climaco A, et al. Risk factors for multidrugresistant organisms among deceased organ donors. Am J Transplant. 2019; 19: 2468-2478.

52. Procaccio F, Masiero L, Vespasiano F, Grossi PA, Gagliotti C, Pantosti A, et al. Organ donor screening for carbapenem-resistant Gram-negative bacteria in italian intensive care units: The drin study. Am J Transplant. 2020; 20: 262-273.

53. Bassetti M, Carnelutti A, Castaldo N, Peghin M. Important new therapies for methicillinresistant. Expert Opin Pharmacother. 2019; 20: 2317-2334.

54. Noval M, Banoub M, Claeys KC, Heil E. The battle is on: New beta-lactams for the treatment of multidrug-resistant Gram-negative organisms. Curr Infect Dis Rep. 2020; 22: 1.

55. Martin Mateos R, Albillos A. Sepsis in patients with cirrhosis awaiting liver transplantation: New trends and management. Liver Transpl. 2019; 25: 1700-1709.

56. Zuccaro V, Columpsi P, Sacchi P, Lucà MG, Fagiuoli S, Bruno R. Antibiotic stewardship and empirical antibiotic treatment: How can they get along? Dig Liver Dis. 2017; 49: 579-584.

57. Hand J, Patel G. Antimicrobial stewardship in transplant patients. Curr Opin Organ Transplant. 2019; 24: 497-503.

58. Safdar N, Said A, Lucey MR. The role of selective digestive decontamination for reducing infection in patients undergoing liver transplantation: A systematic review and meta-analysis. Liver Transpl. 2004; 10: 817-827.

59. Gurusamy KS, Nagendran M, Davidson BR. Methods of preventing bacterial sepsis and wound complications after liver transplantation. Cochrane Database Syst Rev. 2014: CD006660.

60. Katchman E, Marquez M, Bazerbachi F, Grant D, Cattral M, Low CY, et al. A comparative study of the use of selective digestive decontamination prophylaxis in living-donor liver transplant recipients. Transpl Infect Dis. 2014; 16: 539-547.

61. Kelly CR, Ihunnah C, Fischer M, Khoruts A, Surawicz C, Afzali A, et al. Fecal microbiota transplant for treatment of clostridium difficile infection in immunocompromised patients. Am J Gastroenterol. 2014; 109: 1065-1071.

62. Tavoukjian $\mathrm{V}$. Faecal microbiota transplantation for the decolonization of antibiotic-resistant bacteria in the gut: A systematic review and meta-analysis. J Hosp Infect. 2019; 102: 174-188.

63. Saha S, Tariq R, Tosh PK, Pardi DS, Khanna S. Faecal microbiota transplantation for eradicating carriage of multidrug-resistant organisms: A systematic review. Clin Microbiol Infect. 2019; 25: 958-963.

64. Battipaglia G, Malard F, Rubio MT, Ruggeri A, Mamez AC, Brissot E, et al. Fecal microbiota transplantation before or after allogeneic hematopoietic transplantation in patients with hematologic malignancies carrying multidrug-resistance bacteria. Haematologica. 2019; 104: 1682-1688. 
65. Bajaj JS, Fagan A, Gavis EA, Kassam Z, Sikaroodi M, Gillevet PM. Long-term outcomes of fecal microbiota transplantation in patients with cirrhosis. Gastroenterology. 2019; 156: 19211923.e1923.

66. Bajaj JS, Kassam Z, Fagan A, Gavis EA, Liu E, Cox IJ, et al. Fecal microbiota transplant from a rational stool donor improves hepatic encephalopathy: A randomized clinical trial. Hepatology. 2017; 66: 1727-1738.

67. Defilipp Z, Bloom PP, Torres Soto M, Mansour MK, Sater MRA, Huntley MH, et al. Drugresistant. N Engl J Med. 2019; 381: 2043-2050.

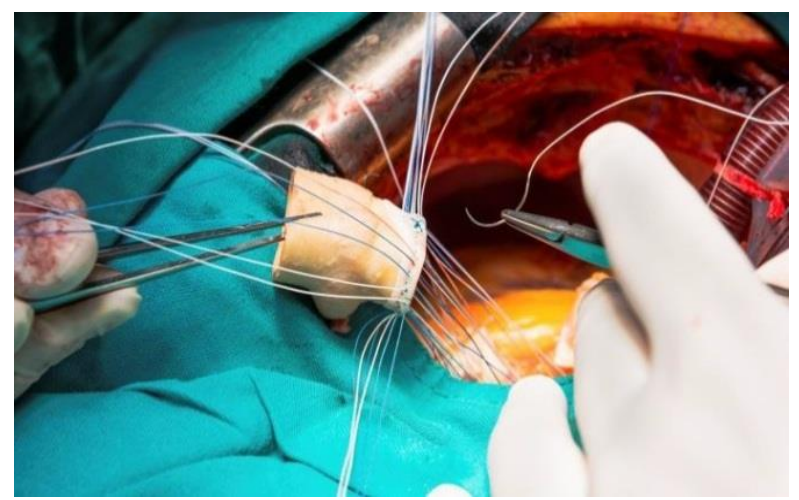

Enjoy OBM Transplantation by:

1. Submitting a manuscript

2. Joining in volunteer reviewer bank

3. Joining Editorial Board

4. Guest editing a special issue

For more details, please visit:

http://www.lidsen.com/journals/transplantation 